UCRL-JC-122230

PREPRINT

CONF $-9603 / 19--2$

\title{
Evaluation of the Discrete Complex-Image Method for a NEC-Like Moment-Method Solution
}

\author{
G. J. Burke
}

This paper was prepared for submittal to

The 12th Annual Review of Progress in Applied Computational Electromagnetics

Monterey, CA

March 18-22, 1996

January 5, 1996

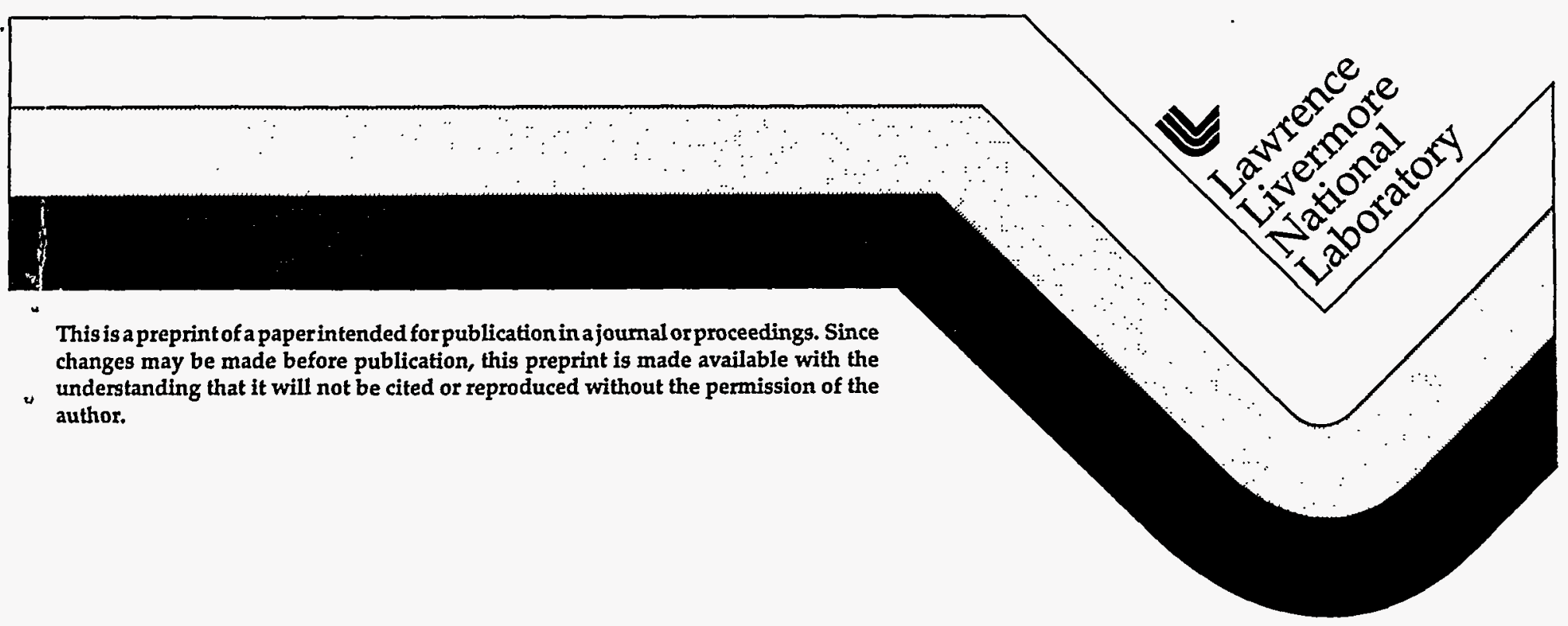




\section{DISCLAIMER}

This document was prepared as an account of work sponsored by an agency of the United States Government. Neither the United States Government nor the University of California nor any of their employees, makes any warranty, express or implied, or assumes any legal liability or responsibility for the accuracy, completeness, or usefulness of any information, apparatus, product, or process disclosed, or represents that its use would not infringe privately owned rights. Reference herein to any specific commercial product, process, or service by trade name, trademark, manufacturer, or otherwise, does not necessarily constitute or imply its endorsement, recommendation, or favoring by the United States Government or the University of California. The views and opinions of authors expressed herein do not necessarily state or reflect those of the United States Government or the University of California, and shall not be used for advertising or product endorsement purposes. 


\title{
EVALUATION OF THE DISCRETE COMPLEX-IMAGE METHOD FOR A NEC-LIKE MOMENT-METHOD SOLUTION *
}

\author{
G. J. Burke \\ Lawrence Livermore National Laboratory \\ P.O. Box 5504, L-156, Livermore, CA 94550
}

\begin{abstract}
The discrete image approximation for the field of a half-space is tested in the NEC antenna modeling program as an alternative to the interpolation method presently used. The accuracy and speed of the discrete image approximation are examined for varying number of images and approximation contour, and the solution for current is obtained on a horizontal wire approaching the interface.
\end{abstract}

\section{INTRODUCTION}

A common extension to frequency domain moment-method codes is to model structures in a stratified medium or at least near a homogeneous ground. The solution then requires the Green's function for the stratified medium or half-space, which in its exact form involves an integral over an infinite spectrum of waves $[1,2]$. The moment-method solution generally requires many evaluations of the Green's function for numerical integration over sources, so evaluation time and accuracy become critical. A number of methods have been used in this evaluation, including integration over the real radial wavenumber or on a contour deformed to the steepest descent path [3]. Linear filters have been very effective for lossy media [4]. Lindell [5] has converted the spectral integrals of Sommerfeld into integrals over an image distributed in complex space. While this solution remains exact, it still requires numerical integration over the distributed images, although the integrals may be more easily evaluated than the spectral form. The code NEC [6] uses an interpolation method for a half-space [7]. The Sommerfeld integrals are evaluated numerically on contours deformed to accelerate convergence [8]. The values are then transformed using the approximate quasistatic or asymptotic behavior of the fields to remove rapid variations, and are stored in tables from which the required values are obtained by interpolation. Alternatively, a "model" containing functions from the asymptotic solution and variable parameters is fit to the computed values in a technique known as model-based parameter estimation [9]. These interpolation methods are fast, accurate and reasonably simple when source and evaluation points are in the same medium, but can get complicated for interactions across interfaces.

More recently a simple approximation of the Green's function for a stratified medium or half-space has been developed in terms of a sum of discrete images in complex space [10]. This method has been applied to the solution of microstrip problems $[11,12]$ and to antennas over a homogeneous ground [13] using the mixed-potential form of the integral equation. In this paper results of using the discrete image method in a solution with continuous current expansion and

* Work performed under the auspices of the U. S. Department of Energy by the Lawrence Livermore National Laboratory under Contract W-7405-Eng-48. 
point matching of the field are reported. The accuracy of the discrete image approximation is investigated, and the speed is compared with the interpolation method in NEC.

\section{THE DISCRETE IMAGE APPROXIMATION FOR A HALF-SPACE}

The discrete image approximation is obtained by numerical processing of the Sommerfeld integrals for the potentials due to a half-space, after first extracting a quasistatic term to improve convergence. The method is outlined here to obtain the field components needed in NEC. The solution for the field of a source in the presence of a half-space can be found in many references, for example $[1,2]$. The geometry of the problem is shown in figure 1 , using the convention that the lower medium is medium 1 and the upper is medium 2. The wavenumbers are $k_{1}=k_{0}\left(\epsilon_{\mathrm{i}}-\right.$ $\left.j \sigma_{1} / \omega \epsilon_{0}\right)^{1 / 2}$ and $k_{2}=k_{0}\left(\epsilon_{2}-j \sigma_{2} / \omega \epsilon_{0}\right)^{1 / 2}$ with $k_{0}=\omega \sqrt{\mu_{0} \epsilon_{0}}$ and $e^{j \omega t}$ time variation assumed. The form of the solution used here is from [2] in terms of the potentials $U_{22}$ and $V_{22}$ which, together with the free-space Green's functions for the source and

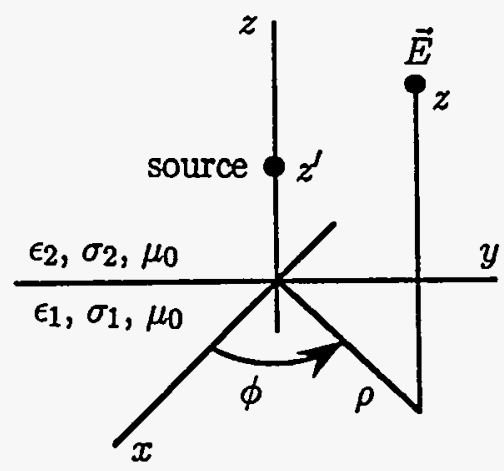

Fig. 1. Geometry for source and evaluation points above a half-space

its image, yield all of the components of electric field in the upper medium due to vertical and horizontal electric dipoles in the upper medium. The potentials involve infinite integrals over the radial component of wavenumber $k_{\rho}$ which can be written in terms of either Bessel or Hankel functions in $k_{\rho}$ as

$$
\begin{aligned}
V_{22} & =2 \int_{0}^{\infty} R_{V}\left(k_{z 2}\right) \frac{e^{-j k_{z 2}\left(z+z^{\prime}\right)}}{k_{z 2}} J_{0}\left(k_{\rho} \rho\right) k_{\rho} d k_{\rho} \\
& =\int_{-\infty}^{\infty} R_{V}\left(k_{z 2}\right) \frac{e^{-j k_{z 2}\left(z+z^{\prime}\right)}}{k_{z 2}} H_{0}^{(2)}\left(k_{\rho} \rho\right) k_{\rho} d k_{\rho} \\
U_{22} & =2 \int_{0}^{\infty} R_{U}\left(k_{z 2}\right) \frac{e^{-j k_{z 2}\left(z+z^{\prime}\right)}}{k_{z 2}} J_{0}\left(k_{\rho} \rho\right) k_{\rho} d k_{\rho} \\
& =\int_{-\infty}^{\infty} R_{U}\left(k_{z 2}\right) \frac{e^{-j k_{z 2}\left(z+z^{\prime}\right)}}{k_{z 2}} H_{0}^{(2)}\left(k_{\rho} \rho\right) k_{\rho} d k_{\rho}
\end{aligned}
$$

where

$$
R_{V}\left(k_{z 2}\right)=\frac{k_{z 2}}{k_{2}^{2} k_{z 1}+k_{1}^{2} k_{z 2}}, \quad R_{U}\left(k_{z 2}\right)=\frac{k_{z 2}}{k_{z 1}+k_{z 2}}
$$

and $k_{z 1}=\left(k_{1}^{2}-k_{\rho}^{2}\right)^{1 / 2}$ and $k_{z 2}=\left(k_{2}^{2}-k_{\rho}^{2}\right)^{1 / 2}$ with $\operatorname{Im}\left(k_{z 1}, k_{z 2}\right) \leq 0$. The basic contours for evaluating these integrals on the real axis are shown in figure 2 as $C_{0}$ for the Bessel function form and $C_{1}$ for the Hankel function form.

As $R_{1}=\left[\rho^{2}+\left(z+z^{\prime}\right)^{2}\right]^{1 / 2}$ becomes small the integrals in (1) converge more slowly, leading to a $R_{1}^{-1}$ singularity in the integrals. The quasistatic term containing this singularity can be extracted by subtracting the constant limits that $R_{V}$ and $R_{U}$ approach as $k_{\rho}$ or $k_{z 2}$ become large. 
a)

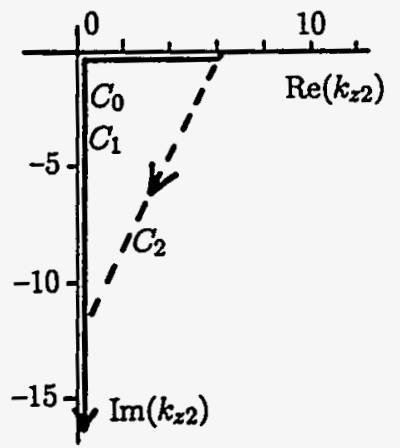

b)

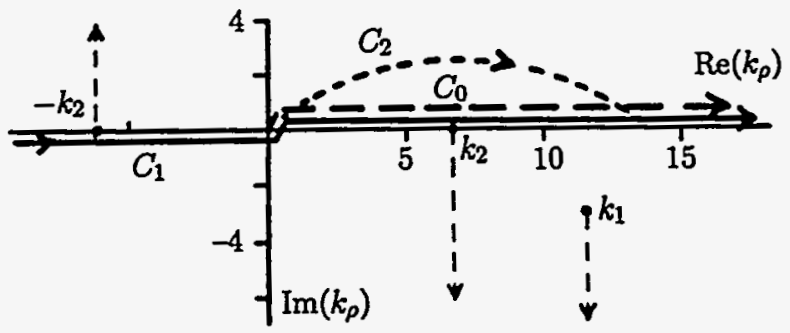

Fig. 2. Contours for evaluation of the Sommerfeld integrals: a) contours in the $k_{z 2}$ plane, b) contours in the $k_{p}$ plane, with branch cuts from $k_{1}$ and $k_{2}$.

The remainders of the potentials after subtracting the quasistatic terms are

$$
\begin{aligned}
& v_{22}=2 \int_{0}^{\infty} R_{v}\left(k_{z 2}\right) \frac{e^{-j k_{z 2}\left(z+z^{\prime}\right)}}{k_{z 2}} J_{0}\left(k_{\rho} \rho\right) k_{\rho} d k_{\rho} \\
& u_{22}=2 \int_{0}^{\infty} R_{u}\left(k_{z 2}\right) \frac{e^{-j k_{z 2}\left(z+z^{\prime}\right)}}{k_{z 2}} J_{0}\left(k_{\rho} \rho\right) k_{\rho} d k_{\rho}
\end{aligned}
$$

where

$$
R_{v}\left(k_{z 2}\right)=\frac{k_{z 2}}{k_{2}^{2} k_{z 1}+k_{1}^{2} k_{z 2}}-\frac{1}{k_{1}^{2}+k_{2}^{2}}, \quad R_{u}\left(k_{z 2}\right)=\frac{k_{z 2}}{k_{z 1}+k_{z 2}}-\frac{1}{2}
$$

The extracted quasistatic terms can be combined with the free space Green's function for the image by applying the Sommerfeld identity,

$$
\int_{0}^{\infty} \frac{e^{-j k_{z 2}\left(z+z^{\prime}\right)}}{k_{z 2}} J_{0}\left(k_{\rho}\right) k_{\rho} d k_{\rho}=\frac{e^{-j k_{2} R_{1}}}{R_{1}}
$$

The equations for the electric field components are then

$$
\begin{aligned}
& E_{\rho}^{V}=\frac{-j \omega I \ell_{\mu_{0}}}{4 \pi k_{2}^{2}} \frac{\partial^{2}}{\partial \rho \partial z}\left(G_{22}+\frac{k_{1}^{2}-k_{2}^{2}}{k_{1}^{2}+k_{2}^{2}} G_{21}\right)+S_{\rho}^{V} \\
& E_{z}^{V}=\frac{-j \omega I \ell \mu_{0}}{4 \pi k_{2}^{2}}\left(\frac{\partial^{2}}{\partial z^{2}}+k_{2}^{2}\right)\left(G_{22}+\frac{k_{1}^{2}-k_{2}^{2}}{k_{1}^{2}+k_{2}^{2}} G_{21}\right)+S_{z}^{V} \\
& E_{\rho}^{H}=\frac{-j \omega I \ell \mu_{0}}{4 \pi k_{2}^{2}}\left(\frac{\partial^{2}}{\partial \rho^{2}}+k_{2}^{2}\right)\left(G_{22}-\frac{k_{1}^{2}-k_{2}^{2}}{k_{1}^{2}+k_{2}^{2}} G_{21}\right) \cos \phi+S_{\rho}^{H} \\
& E_{\phi}^{H}=\frac{j \omega I \ell \mu_{0}}{4 \pi k_{2}^{2}}\left(\frac{1}{\rho} \frac{\partial}{\partial \rho}+k_{2}^{2}\right)\left(G_{22}-\frac{k_{1}^{2}-k_{2}^{2}}{k_{1}^{2}+k_{2}^{2}} G_{21}\right) \sin \phi+S_{\phi}^{H} \\
& E_{z}^{H}=\frac{-j \omega I \ell \mu_{0}}{4 \pi k_{2}^{2}} \frac{\partial^{2}}{\partial \rho \partial z}\left(G_{22}-\frac{k_{1}^{2}-k_{2}^{2}}{k_{1}^{2}+k_{2}^{2}} G_{21}\right) \cos \phi-S_{\rho}^{V} \cos \phi
\end{aligned}
$$

where the subscripts on $E$ or $S$ indicate the cylindrical component of the field and the superscript indicates a vertical electric dipole $(\mathrm{V})$ or horizontal dipole on along the $x$ axis $(\mathrm{H})$. $G_{22}$ and $G_{21}$ 
are the free space Green's functions for the source and its image, $G_{22}=\exp \left(-j k_{2} R_{2}\right) / R_{2}$ and $G_{21}=\exp \left(-j k_{2} R_{1}\right) / R_{1}$ with $R_{\frac{1}{2}}=\left[\rho^{2}+\left(z \pm z^{\prime}\right)^{2}\right]^{1 / 2}$. The final terms, containing the remaining Sommerfeld integrals, are

$$
\begin{aligned}
S_{\rho}^{V} & =\frac{-j \omega I \ell \mu_{0}}{4 \pi k_{2}^{2}} \frac{\partial^{2}}{\partial \rho \partial z} k_{1}^{2} v_{22} \\
S_{z}^{V} & =\frac{-j \omega I \ell \mu_{0}}{4 \pi k_{2}^{2}}\left(\frac{\partial^{2}}{\partial z^{2}}+k_{2}^{2}\right) k_{1}^{2} v_{22} \\
S_{\rho}^{H} & =\frac{-j \omega I \ell \mu_{0}}{4 \pi} \cos \phi\left(\frac{\partial^{2} v_{22}}{\partial \rho^{2}}+u_{22}+G_{21}-\frac{2 k_{2}^{2}}{k_{1}^{2}+k_{2}^{2}} G_{21}\right) \\
S_{\phi}^{H} & =\frac{j \omega I \ell \mu_{0}}{4 \pi} \sin \phi\left(\frac{1}{\rho} \frac{\partial v_{22}}{\partial \rho}+u_{22}+G_{21}-\frac{2 k_{2}^{2}}{k_{1}^{2}+k_{2}^{2}} G_{21}\right) .
\end{aligned}
$$

The final $G_{21}$ terms are included in (6c) and (6d) to complete the field of the quasistatic image in (5) as the field of a source in free space. After subtracting the quasistatic terms, the remaining integrals $u_{22}$ and $v_{22}$ remain finite as $R_{1}$ goes to zero, while the field components in equations (6) have $1 / R_{1}$ singularities from the derivatives of $v_{22}$. The advantage of extracting the quasistatic components for the discrete image approximation is that the functions $R_{u}\left(k_{z 2}\right)$ and $R_{v}\left(k_{z 2}\right)$ in equation (3) decay as $k_{z 2}^{-2}$ for large $k_{z 2}$, and these decaying functions are better suited to approximation by a sum of exponentials than are $R_{U}$ and $R_{V}$ which become constant. The quasistatic terms are also subtracted in the interpolation method used in NEC, where the singular remainders in equation (6) are multiplied by $R_{1}$ so that they can easily be approximated with linear or quadratic interpolation. Alternatively, the singularities in equations (6) can be approximated [6] and also the next constant terms can be obtained. For example, in $S_{\rho}^{H}$

$$
\begin{aligned}
\frac{\partial^{2} v_{22}}{\partial \rho^{2}} \approx & \frac{k_{2}^{2}\left(k_{2}^{2}-k_{1}^{2}\right)}{\left(k_{1}^{2}+k_{2}^{2}\right)^{2}}\left(\frac{\sin \alpha}{1+\sin \alpha}\right) \frac{1}{R_{1}}+ \\
& \frac{j k_{2}^{2}\left(k_{1}^{4}+k_{1}^{3} k_{2}-k_{1}^{2} k_{2}^{2}-2 k_{1} k_{2}^{3}-2 k_{2}^{4}\right)}{3\left(k_{1}+k_{2}\right)\left(k_{1}^{2}+k_{2}^{2}\right)^{2}}+\frac{j k_{1}^{4} k_{2}^{4} C_{k}}{2\left(k_{1}^{2}-k_{2}^{2}\right)\left(k_{1}^{2}+k_{2}^{2}\right)^{5 / 2}}
\end{aligned}
$$

for small $R_{1}$, where

$$
C_{k}=\tan ^{-1}\left(\frac{2\left(k_{1}-k_{2}\right)\left(k_{1}^{2}-k_{1} k_{2}+k_{2}^{2}\right) \sqrt{k_{1}^{2}+k_{2}^{2}}}{2 k_{1}^{4}-4 k_{1}^{3} k_{2}+5 k_{1}^{2} k_{2}^{2}-4 k_{1} k_{2}^{3}+2 k_{2}^{4}}\right)
$$

with $\operatorname{Im}\left(C_{k}\right) \leq 0$ and $\sin \alpha=\left(z+z^{\prime}\right) / R_{1}$.

For the discrete image approximation $R_{u}\left(k_{z 2}\right)$ and $R_{v}\left(k_{z 2}\right)$ are approximated with a sum of exponential functions in $k_{z 2}$ by means of the Prony [i4] or Matrix Pencil [15] methods. Since these methods require equally spaced samples in a real variable, the substitution $k_{z 2}=k_{2}\left[t_{a}+t\left(t_{b}-t_{a}\right)\right]$ is made. Applying the Matrix Pencil method with equally spaced samples over $0 \leq t \leq 1$ yields the exponential approximation over the range $k_{z 2} \in\left(k_{2} t_{a}^{\prime}, k_{2} t_{b}\right)$ as

$$
R_{v}(t) \approx \sum_{i=1}^{N_{t}} A_{i} e^{B_{i} t}, \quad R_{v}\left(k_{z 2}\right) \approx \sum_{i=1}^{N_{t}} a_{i} e^{b_{i} k_{z 2}}
$$


with $a_{i}=A_{i} e^{-B_{i} t_{a} /\left(t_{b}-t_{a}\right)}$ and $b_{i}=B_{i} /\left[k_{2}\left(t_{b}-t_{a}\right)\right]$. When the exponential approximation of $R_{v}\left(k_{z 2}\right)$ is substituted into (2) the integral for each term can be evaluated using the Sommerfeld identity (4) to get a sum of discrete images

$$
v_{22} \approx \sum_{i=1}^{N_{t}} a_{i} \frac{e^{-j k_{2} R_{i}}}{R_{i}}
$$

with $R_{i}=\left[\rho^{2}+\left(z+z^{\prime}+j b_{i}\right)^{2}\right]^{1 / 2}$. Thus $v_{22}$, and similarly $u_{22}$ are approximated by a sum of free-space Green's functions for images in complex space.

In applying the discrete image method a contour similar to $C_{2}$ in figure 2 is usually chosen for the approximation [10]. Hence, $t_{a}=1$ and $t_{b}$ is a negative imaginary value. This deformation from $C_{0}$ yields a linear path in the $k_{z 2}$ plane and also avoids surface-wave poles that would occur for a stratified medium. It also moves the path further from the Zenneck pole in $R_{V}$, making the approximation easier.

\section{NUMERICAL RESULTS}

The accuracy of the discrete image approximation was tested against the numerical evaluation routines in SOMNEC [6] after reducing the error limits in the Romberg adaptive integration by two orders of magnitude and increasing the accuracy of the Bessel and Hankel functions. With these changes the relative error in the integration seems to be around $10^{-6}$ to $10^{-7}$.

The error from the discrete image approximation for $v_{22}$ is shown in figure $3 \mathrm{a}$ for a lossy ground with the number of images $N_{t}$ varied. The matrix pencil method was used with $N_{s}=300$ samples and $t_{b}=-j 10 . N_{t}=12$ was the maximum number of terms that could be obtained from the matrix pencil method with the tolerance in the singular-value decomposition set to $10^{-10}$. The approximation is seen to converge rapidly in the region of $0.1<R_{1} / \lambda_{0}<1$. The error with varying $t_{b}$ is shown in figure $3 \mathrm{~b}$, where $N_{t}$ was always the maximum returned by matrix pencil. The increased error for small $R_{1}$ is due to the truncation of the approximation contour at $k_{2} t_{b}$ as the integrand converges more slowly, so a larger $t_{b}$ reduces the error. The use of the Sommerfeld identity for equation (8) implies an integration contour to infinity, but the error is uncontrolled beyond $k_{2} t_{b}$ and increases as the integrand decays. The increased error in figure $3 \mathrm{~b}$ for large $R_{1}$ and large $t_{b}$ is due to insufficient sampling, and would be reduced with a larger $N_{s}$ at the cost of increased time for determining the image parameters. Figure $3 \mathrm{c}$ shows the error with varying $t_{b}$ for grazing incidence along the ground. The error due to truncation at $t_{b}$ occurs sooner as $R_{1}$ is decreased than for points off the interface, since the integrand decays more slowly without the $e^{-j k_{x 2}\left(z+z^{\prime}\right)}$ term. In numerical integration the Hankel function form of equation (1) could be used in this case, with the integration contour deformed downward to a steepest descent path. The contour $C_{2}$ used in the discrete image approximation is more nearly optimum for large $\left(z+z^{\prime}\right) / \rho$. For large $R_{1}$ the error increases rapidly, apparently from difficulty in approximating $R_{v}$ near the Zenneck pole near $k_{z 2}=k_{2}$. A small $t_{b}$ reduces the error for large $R_{1}$ with increased error for small $R_{1}$. Figure $3 \mathrm{~d}$ shows the same result as $3 \mathrm{c}$ but for dielectric ground. In this case the error increases more rapidly for large $R_{1}$, perhaps due to the difficulty in approximating the lateral wave. Although the lateral wave, with wavenumber $k_{1}$, must be synthesized from exponentials in $k_{2}$ the approximation is successful for a number of cycles determined by the number of discrete image terms. For example, the lateral wave was approximated to about $R_{1} / \lambda=0.7$ with 5 
a)

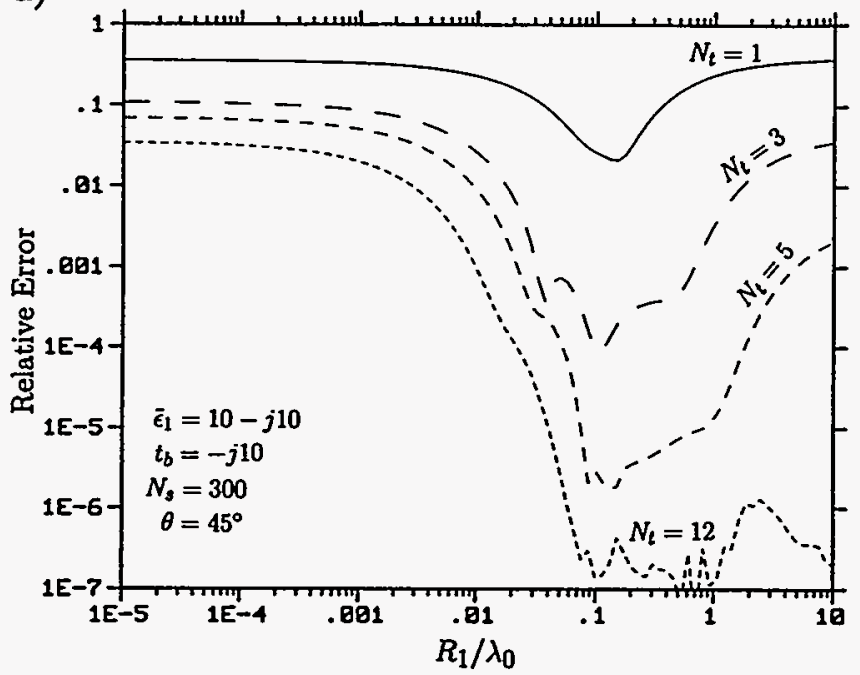

c)

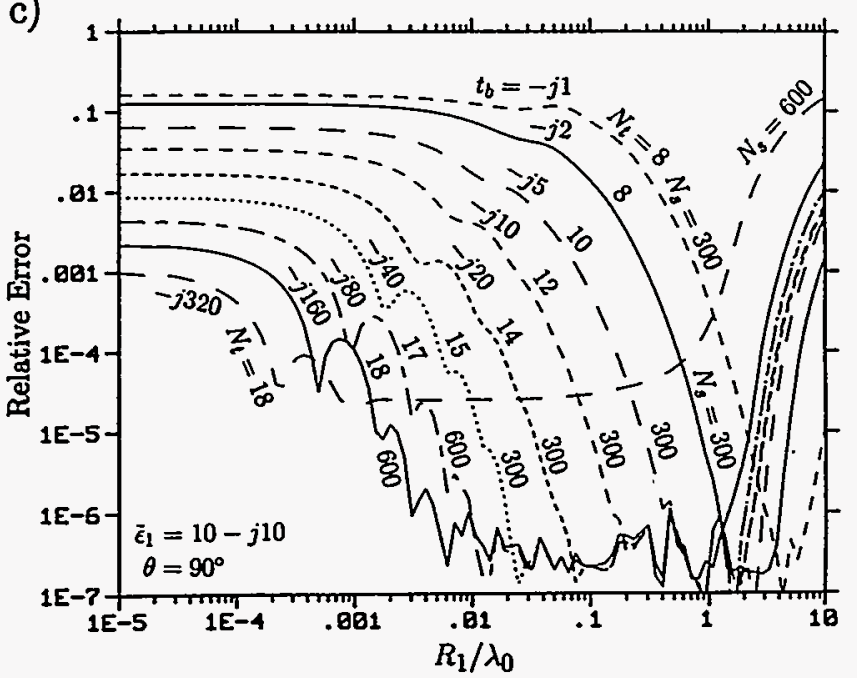

b)

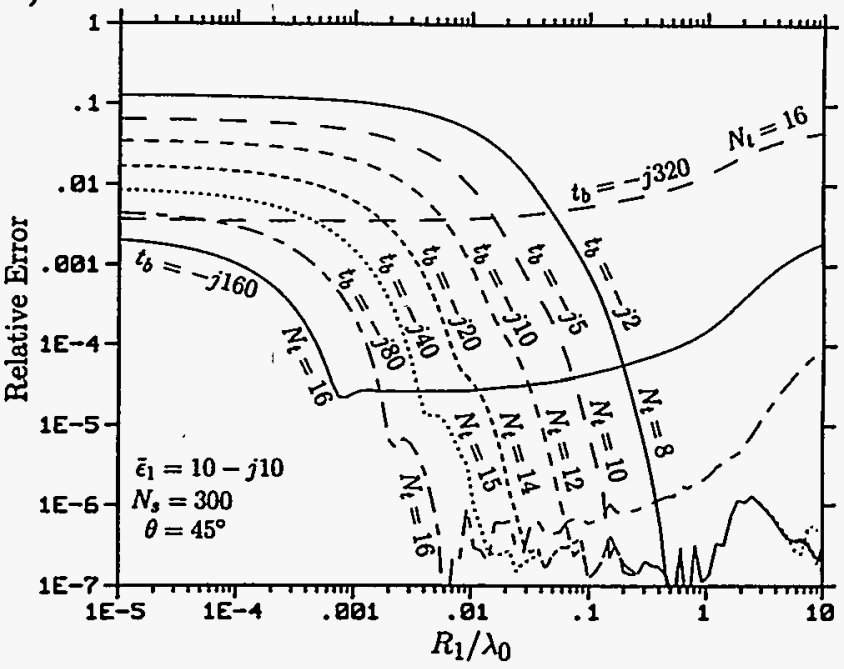

d)

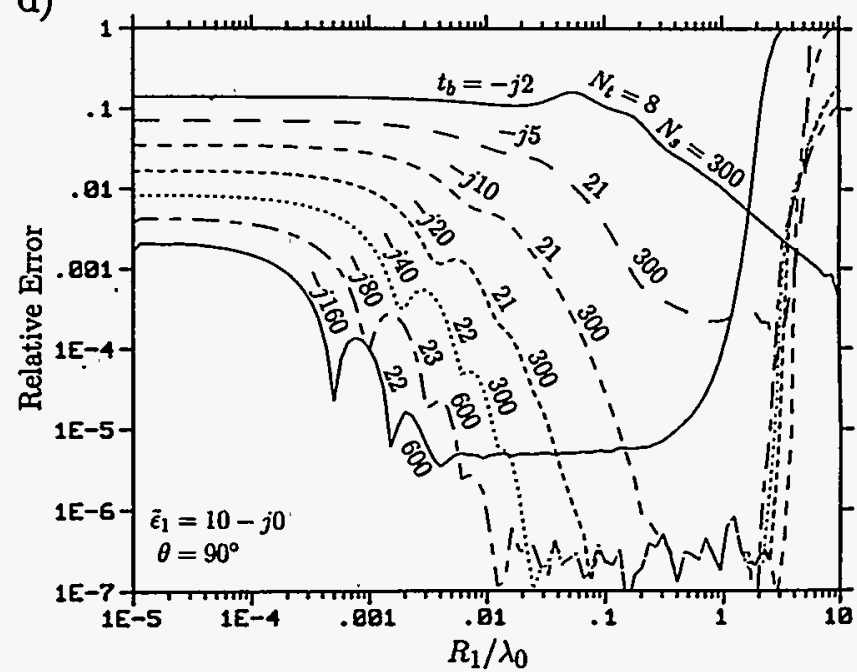

Fig. 3. Error in the discrete image approximation of $v_{22}$ with $N_{t}$ images and $N_{s}$ samples. The ground permittivity is $\tilde{\epsilon}=\epsilon_{1}-j \sigma / \omega \epsilon_{0}$ and $\theta=\tan ^{-1}\left[\rho /\left(z+z^{\prime}\right)\right]$.

images, to $R_{1} / \lambda=1.3$ with 10 images and to $R_{1} / \lambda=3$ with 22 images. Beyond these distances the approximation diverged rapidly.

Figure 4 shows the relative error in approximating $\partial^{2} v_{22} / \partial \rho^{2}$ for the $E_{\rho}^{H}$ field component needed to model a horizontal wire over ground. The increased error over the approximation for $v_{22}$ shows that the $1 / R_{1}$ singularity in this second derivative is not contained in the discrete image approximation. When integrated over a filament of current, this $1 / R_{1}$ term contributes to the log singularity in the field parallel to the filament, although the importance of it relative to the integral of the quasistatic image terms depends on the coefficients multiplying the terms. If necessary the approximations from equation (7), shown as curves $A_{1}$ and $A_{2}$ in figure 4, can be used to reduce the error.

A version of NEC-2 has been set up to use the discrete image approximation instead of interpolation for arbitrary wire structures above ground. As an initial test, a horizontal $\lambda / 2$ 


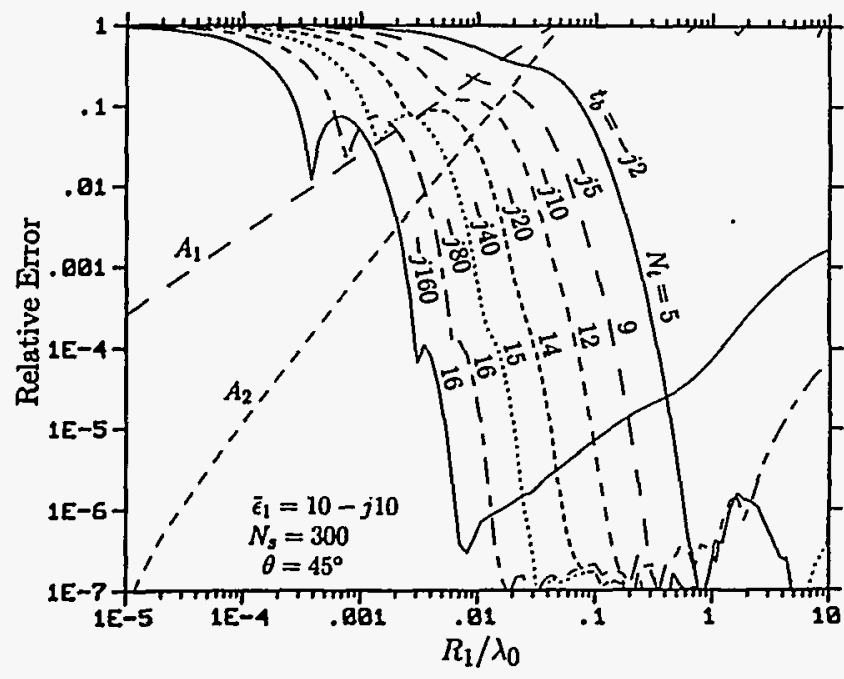

Fig. 4. Error in approximating $\partial^{2} v_{22} / \partial \rho^{2}$ for varying contour limit $t_{b}$. Curves $A_{1}$ and $A_{2}$ are quasistatic approximations from equation (7) with the first term $\left(1 / R_{1}\right)$ and all terms, respectively.

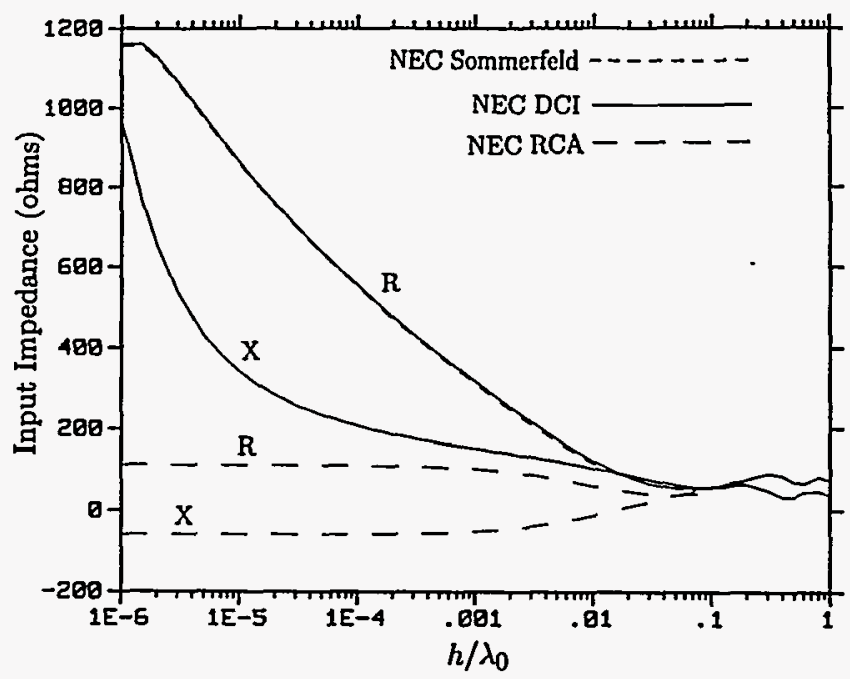

Fig. 5. Input impedance of $a \lambda_{0} / 2$ dipole with radius $10^{-7} \lambda_{0}$ at height $h$ above the ground, comparing results of NEC Sommerfeld/interpolation, discrete image (DCI) and Fresnel reflection coefficient (RCA).

dipole oyer ground was modeled, since it was expected to be a more difficult test than a vertical wire. Results with varying numbers of images, with and without the quasistatic approximation in equation (7), were in excellent agreement with the standard NEC-2. Figure 5 shows the result with one discrete image for $u_{22}$ and zero discrete images for $v_{22}$, and the agreement is very good. With three images for each potential the noticeable difference between the curves essentially disappears. If the single discrete image for $u_{22}$ is dropped, using only quasistatic terms, the error in impedance for small $h / \lambda_{0}$ is about twenty percent, and the wiggles between 0.1 and 1 in $h / \lambda_{0}$ are not tracked well. Hence it appears that for the horizontal $\lambda / 2$ dipole close to the ground, the quasistatic images are most important, and a relatively crude approximation of the remainder is sufficient. Other structures, such as a long horizontal wire or top loaded monopole may put more demand on accuracy of the discrete image approximation and will be investigated in the future. The result of using the Fresnel plane-wave reflection coefficient is also shown in figure 5 and, as expected, is accurate for $h / \lambda$ greater than about 0.1 .

The CPU time for evaluating all field components in equation (6) by the discrete image method on a DEC 3000/400 computer was $89 \mu$ s with 3 images for each potential and $283 \mu \mathrm{s}$ with 10 images. For comparison, the time for a single evaluation by the NEC-2 interpolation is 7.2 $\mu \mathrm{s}$. The difference is mainly due to the number of complex exponentials that must be evaluated. The time to compute the parameters of the discrete image approximation is proportional to $N_{s}^{3}$, and ranged from $0.04 \mathrm{~s}$ with 50 samples to $58 \mathrm{~s}$ with 600 samples, while the time to generate the NEC-2 interpolation table was $1.3 \mathrm{~s}$.

\section{CONCLUSION}

The discrete image approximation can provide a simple and accurate approximation for the field over ground for separations of image and evaluation points in the range of about 0.01 to several wavelengths. The limit for large distance is most restrictive along the interface where surface and lateral waves are significant, but asymptotic approximations can fill the gap to infinity. 
Accuracy decreases for very small distances due to truncation of the approximation contour, but the errors become less significant relative to the quasistatic terms. The approximation contour and number of images can be adjusted to optimize the approximation for a given range of distances and accuracy. The discrete images provide a simple and highly compact representation for the field. The code to obtain the image parameters using the matrix pencil method was about 24,000 lines using the LAPAC routines, but simpler routines should be available.

The discrete image approximation can also be used when source and evaluation points are on opposite sides of the interface, but its usefulness seems much more limited since the image parameters must be recomputed for each new source location, or for each new evaluation point [10]. It still might be usable in a moment-method with efficient "bookkeeping" in filling the matrix.

\section{References}

[1] A.Sommerfeld, "Über die Ausbreitung der Wellen in der drahtlosen Telegraphie," Ann. Physik, Vol. 28, p. 665, 1909.

[2] A. Baños, Dipole Radiation in the Presence of a Conducting Half-Space, Pergamon Press, New York, 1966.

[3] K. A. Michalski, "On the efficient evaluation of integrals arising in the Sommerfeld halfspace problem," IEE Proc., Vol. 132, Pt. H, No. 5, Aug. 1985.

[4] O. Koefoed, D. P. Gosh and G. J. Polman, "Computation of type curves for electromagnetic depth sounding with a horizontal transmitting coil by means of a digital linear filter," Geophysical Prospecting, Vol. 20, pp. 406-420, 1972.

[5] I. V. Lindell and E. Alanen, "Exact image theory for the Sommerfeld half-space problem - Pt. 1: Vertical magnetic dipole," IEEE Trans., AP-32, pp. 126-132, 1984.

[6] G. J. Burke and A. J. Poggio, "Numerical Electromagnetics Code (NEC) - Method of Moments,", Lawrence Livermore National Laboratory Rept. UCID-18834, January 1981.

[7] J. N. Brittingham, E. K. Miller and J. T. Okada, SOMINT: An Improved Model for Studying Conducting Objects Near Lossy Half-Spaces, UCRL-52423, Lawrence Livermore National Laboratory, CA, February 24, 1978.

[8] R. J. Lytle and D. L. Lager, Numerical Evaluation of Sommerfeld Integrals, UCRL-51688, Lawrence Livermore National Laboratory, CA, October 23, 1974.

[9] G. J. Burke, Numerical Electromagnetics Code - NEC-4, UCRL-MA-109338, Lawrence Livermore . National Laboratory, CA, January 1992.

[10] D. G. Fang, J. J. Yang and G. Y. Delisle, "Discrete image theory for horizontal electric dipoles in a multilayered medium," IEE Proc., Vol. 135, Pt. H, No. 5, Oct. 1988.

[11] Y. L. Chow, J. J. Yang, D. G. Fang and G. E. Howard, "A Closed-Form Spatial Green's Function for the Thick Microstrip Substrate," IEEE Trans. MTT, Vol. 39, No. 3, March 1991.

[12] M. I. Aksun and R. Mitra, "Derivation of Closed-Form Green's Functions for a General Microstrip Geometry," IEEE Trans. MTT, Vol. 40, No. 11, Nov. 1992.

[13] R. M. Shubair and Y. L. Chow, "A Simple and Accurate Complex Image Interpretation of Vertical Antennas Present in Contiguous Dielectric Half-Spaces," IEEE Trans. Ant. and Propag., Vol. 41, No. 6, June 1993.

[14] F. B. Hildebrand, Introduction to Numerical Analysis, McGraw Hill Co., New York, pp. 378-382, 1956.

[15] T. K. Sarkar and O. Pereira, "Using the Matrix Pencil Method to Estimate the Parameters of a Sum of Complex Exponentials," IEEE Antennas and Propagation Magazine, Vol. 37, No. 1, February 1995. 
- . 


$$
1
$$

